\title{
Familia y escuela: dos contextos comprometidos con la formación en ciclo III de la educación básica*
}

\author{
Alexandra Patricia Pedraza** \\ Claudia Patricia Salazar ${ }^{* * *}$ \\ Arsenio Enrique Robayo*** \\ Emerson Arturo Moreno
}

Recibido: 14 de noviembre de 2016 - Aprobado: 13 de marzo de 2017

\section{Resumen}

El presente artículo se circunscribe en torno a las reflexiones que sobre las percepciones de la relación familia y escuela tienen niños que cursan el ciclo III de educación básica. Se pretende profundizar en los diferentes matices de las relaciones entre padres y docentes y en cómo dichas interacciones fortalecen o contradicen el éxito escolar.

La escuela es una institución cuyo objeto esencial es la formación del ser humano, pero dicha misión no puede consolidarse sin la participación proactiva de la institución familiar ni sin el apoyo decidido del estado y la sociedad. En torno a dichos cuestionamientos y a lo que manifiestan los estudiantes entrevistados, se teje una red de conceptos que tratan de dilucidar las coyunturas que se presentan al interior de los hogares y los vaivenes que caracterizan a la compleja relación familia y escuela.

Palabras clave: educación; participación; familia-escuela; éxito escolar

* Artículo de reflexión que se enmarca en el macroproyecto investigativo: "Rol de la familia en las estrategias de retención estudiantil de educación básica y media" de la Maestría en Asesoría Familiar y Gestión de Programas para la Familia, del Instituto de la Familia de la Universidad de la Sabana en Colombia. DOI: http://dx.doi.org/10.15332/ s0120-8454.2017.0091.02

** Psicóloga de la Universidad Santo Tomás, especialista en Gerencia Educativa y Magíster en Educación de la Universidad de la Sabana. Docente e investigadora en la Universidad de la Sabana, Universidad el Bosque, Universidad Militar Nueva Granada y Fundación Universitaria del Área Andina, Colombia. Investigadora, docente y asistente de coordinación en los niveles de educación preescolar, básica, media y superior. Dirección postal: Campus del Puente del Común, Km. 7 Autopista Norte. Chia, Cundinamarca, Colombia. Correo electrónico: alex_pedrazaor@hotmail.com. ORCID: http:// orcid.org/0000-0002-2164-0854

*** Licenciada en Educación Preescolar de la Fundación Universitaria Monserrate. Docente del Programa Volver a la Escuela (Aula Procesos Básicos). Participación en la implementación de la propuesta pedagógica para las aulas del programa Volver a la Escuela de Bogotá, Secretaría de Educación y la Fundación Dividendo Por Colombia, 2012. Correo electrónico: claudia2008cps@hotmail.com

**** Magister en Educación de la Universidad Javeriana, especialista en Gerencia Social de la Escuela Superior de Administración Pública. Docente y asesor de investigación Fundación Universitaria Juan de Castellanos Tunja (Boyacá). Directivo docente de la Secretaría de Educación de Bogotá D.C. Correo electrónico: arrobacero@gmail.com

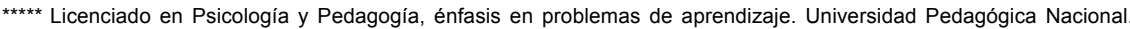
Normalista superior de la Escuela Superior Nuestra Señora de la Paz. Docente, psicopedagogo y orientador de la Secretaría de Educación, Bogotá D.C. Correo electrónico: emartur21@hotmail.com 


\title{
Family and School: two environments commited to development by the IIIrd cycle on the public education system*
}

\author{
Alexandra Patricia Pedraza** \\ Claudia Patricia Salazar*** \\ Arsenio Enrique Robayo ${ }^{* * *}$ \\ Emerson Arturo Moreno ${ }^{* * * * *}$
}

\section{A bstract}

The following article is about diverse reflections on the perceptions in the relation that both, family and school have in children of cycle III of basic education. Thus, it seeks to deeper into the different nuances of the relationships between parents and teachers and how these interactions may strengthen or contradict school success. The school is an institution whose essential object is the formation of the human being; however, this mission cannot be consolidated without the proactive participation of the family institution, or without the determined support of the state and society. So, around these questions and what the students said in different interviews, a network of concepts that try to elucidate the conjunctures that appear inside the homes and the fluctuations that characterize the complex family and school relationship are woven.

Key words: education; participation; family-school; school success

* Reflection article that is part of the research macro project: "Role of the family in the student retention strategies of basic and secondary education" of the Master's Degree in Family Counseling and Management of Family Programs, of the Family Institute of the University of La Sabana in Colombia. DOI: http://dx.doi.org/10.15332/s0120-8454.2017.0091.02

** Psychologist from the University of Santo Tomas, specialist in Educational Management and Master's Degree in Education of the University of La Sabana. Professor and researcher at the University of La Sabana, University El Bosque, Military University Nueva Granada and University Foundation of the Andean Area, Colombia. Researcher, teacher and coordination assistant at pre-school, primary, secondary and higher education levels. Mailing address: Campus del Puente del Común, Km. 7 Autopista Norte. Chia, Cundinamarca, Colombia. Email: alex_pedrazaor@hotmail.com. ORCID: http:// orcid.org/0000-0002-2164-0854

*** Graduated in Pre-school Education from the Monserrate University Foundation. Teacher of the Back to School Program (Basic Processes Classroom). Participation in the implementation of the pedagogical proposal for the Back to School classrooms program in Bogotá, Secretariat of Education and Dividendo Por Colombia Foundation, 2012. E-mail: claudia2008cps@hotmail.com

**** Master's Degree in Education from the Javeriana University, specialist in Social Management from the Higher School of Public Administration. Professor and research adviser University Foundation Juan de Castellanos Tunja (Boyacá). Teacher director of the Secretariat of Education Bogotá D.C. Email: arrobacero@gmail.com

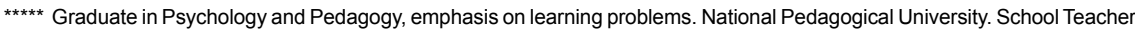
from the Higher School Nuestra Señora de la Paz. Teacher, psychologist and counselor of the Secretariat of Education, Bogotá D.C. Email: emartur21@hotmail.com 


\title{
Famille et école: deux contextes engagés avec la formation dans le cycle III de l'éducation basique*
}

\author{
Alexandra Patricia Pedraza** \\ Claudia Patricia Salazar*** \\ Arsenio Enrique Robayo**** \\ Emerson Arturo Moreno****
}

\section{Résumé}

Cet article se limite aux réflexions que les enfants qui font partie du cycle III de l'éducation basique ont au sujet des perceptions de la relation famille et école. On prétend approfondir dans les différentes teintes des relations entre parents et professeurs, et comment ces interactions renforcent ou contredisent le succès scolaire.

L'école est une institution qui a pour but essentiel la formation de l'être humain, mais cette mission ne peut pas aboutir sans la participation active de la famille, ni sans le soutien décidé de l'état et de la société. Autour des ces questionnements et face à ceux des étudiants, on tisse un réseau de concepts qui essayent d sélucider les situations qui se présentent au sein des ménages et des va-et-vient qui caractérisent la relation complexe entre la famille et l'école.

Mots clés: éducation; participation; famille-école;succès scolaire

* Article de réflexion qui fait partie du macroprojet de recherche: «Le rôle de la famille dans les stratégies de rétention des élèves de l'enseignement primaire et secondaire » du Master en Conseil pour la Famille et Gestion de Programmes pour la Famille, de I'Institut de la Famille de l'Université de la Sabana en Colombie. DOI: http://dx.doi.org/10.15332/ s0120-8454.2017.0091.02

** Psychologue de l'Université Santo Tomás, spécialiste en Gestion Éducative et Magister en Éducation de l'Université de la Sabana. Professeur et chercheur à l'Université de la Sabana, Université El Bosque, Université Militaire Nueva Granada et la Fondation Universitaire de la Zone Andine. Chercheur, enseignante et coordinatrice adjointe au niveau de l'enseignement primaire, secondaire et supérieur. Email: alex_pedrazaor@hotmail.com. ORCID: http://orcid. org/0000-0002-2164-0854

*** Licence en Éducation de la Petite Enfance de la Fondation Universitaire Monserrate. Professeur du Programme Retourner à l'École. Participation dans la mise en œuvre de la proposition pédagogique pour les classes du programme Retourner à l'École de Bogota, Secrétariat de l'Éducation et la Fondation Dividendo Por Colombia, 2012. Email: claudia2008cps@ hotmail.com

**** Magister en Éducation de l'Université Javeriana, spécialiste en Gestion Sociale de l'École Supérieure d'Administration Publique. Professeur et consultant de recherche Fondation Universitaire Juan de Castellanos Tunja, (Boyacá, Colombie). Enseignant et consultant de la Fondation Universitaire Juan de Castellanos Université de Tunja (Boyacá). Directeur et professeur du Secrétariat de l'Éducation de Bogota. Email: arrobacero@gmail.com

***** Licence en Psychologie et Pédagogie, spécialité sur les troubles de l'apprentissage. Université Pédagogique Nationale. Normaliste supérieur de l'École Supérieure Notre-Dame de la Paix. Professeur, psycho-pédagogue scolaire et conseiller du Secrétariat d'Éducation, Bogota. Email: emartur21@hotmail.com 


\section{Introducción}

La afinidad de la familia con la escuela es producto de un proceso de corresponsabilidad en el que tanto los padres como la comunidad educativa desempeñan un papel activo fundamental en el éxito de la formación del estudiante. Corresponde a la familia, ante la exigencia del momento presente, abrirse a nuevos campos educativos para brindar esfuerzos y lograr dar a los hijos elementos básicos en su educación integral.

Familia y escuela constituyen entornos decisivos en la educación del estudiante, por esta razón se ha insistido reiteradamente en la necesidad de una mutua colaboración entre padres y docentes, señalando los logros que se derivan de ella. La familia tiene una función fundamental en el ámbito formativo, dado que frente a los continuos cambios se establece como una obligación, y en un deber, actualizar los procesos formativos que respondan a las urgencias del momento presente. Familia y escuela conforman un dúo de forma destacada: la familia conoce intuitivamente mejor a los hijos y la escuela aporta técnicas científicas para la aprehensión del conocimiento, enmarcadas en todo un proceso pedagógico. La colaboración familia-escuela ha de ser fortalecida de manera que ambas instituciones caminen en una misma dirección y hacia la consecución de metas muy concretas.

La educación familiar hace parte de la unidad familia-escuela, es decir, la relación de la realidad educativa con la familia. En este sentido, hay dos campos de estudio: la educación nace en el seno del ambiente natural y familiar, y de la relación de la familia con la comunidad educativa. Por esta razón, es necesario abordar cómo los factores relacionados con el entorno familiar pueden explicar las diferencias del rendimiento o éxito escolar en mayor medida que otros factores, es decir, que los logros académicos de los estudiantes estarían relacionados con aspectos sociales, culturales, experiencias previas en torno al aprendizaje, actitudes y expectativas presentes en el contexto familiar (Bourdieu y Passeron, 1990).

La misión educativa no podrá desarrollarse de una manera apropiada si no existe un ambiente familiar armonioso. La responsabilidad de los padres es decisiva en la educación de los hijos, pues la familia es el primer medio ambiente necesario y natural para toda educación. Esta idea, que hoy constituye un principio básico en el estudio de la educación, sigue siendo objeto de varias reflexiones teóricas: se pueden ver, entre otros, Díez (1998), Marchesi y Martín (1998), Vila (1998), Sarramona (1999), Molero (2003), Ordóñez (2005), Garreta (2007).

Son considerables las características que se podrían destacar en el ambiente familiar al analizar los beneficios que se darían en el éxito o fracaso escolar de los estudiantes. En el hogar, por ejemplo, los valores que se cultivan se convierten en educación permanente y vivencial, pues las diferentes acciones deben estar enmarcadas en el camino del amor que irá dando el perfil y la identidad de cada uno de sus miembros, dentro del proceso de formación y madurez. En la 
primera etapa de formación del niño se han de tener en cuenta las características culturales y la estructura familiar para considerar la forma de educar a los hijos. Así lo menciona Gimeno (1984) al aclarar que la familia es el medio natural en el que el niño comienza su vida y su aprendizaje:

La familia constituye el medio natural en el que el niño comienza su vida, e inicia su aprendizaje básico a través de una serie de estímulos y de vivencias que le condicionarán profundamente a lo largo de toda su existencia. El clima afectivo de las relaciones internas familiares, así como el nivel cultural, determinan poderosamente la receptividad y la adaptación del alumno en su proceso de escolarización. La estabilidad y equilibrio en la unión de sus padres y las relaciones entre los miembros de la familia definen el clima afectivo, base del proceso de socialización del niño. (p.30)

En este sentido, Suarez (2008) señala que "desde las primeras etapas de la vida, la familia ha de favorecer el despliegue de la identidad personal y la integridad" (p.36) para enfrentar nuevas experiencias, preparar para la madurez, aceptar responsabilidades propias y fomentar la iniciativa individual. La educación dada en el hogar ha de ser íntegra y adecuada, es decir, debe cubrir todas las facetas de la persona, concorde con las circunstancias y características propias de cada una.

Todo trabajo de educación debe partir de las metas propuestas para alcanzar la concepción e ideal de vida. De la formación dada en el hogar dependerá la respuesta que se dé en el medio social. Hay un rol y un papel concreto de los padres en la célula familiar, uno que ostenta autoridad, otro amor y ambos la estabilidad y la seguridad. Aquí está el contexto de una relación de colaboración entre la familia y la escuela, orientada al logro de unos objetivos educativos y a aprendizajes comunes. Las características estructurales y de funcionamiento de la familia, y el ambiente educativo que se lleve en el hogar, son factores que influyen evidentemente en el éxito o fracaso escolar (Ruiz y García, 2001, p.52)

La literatura vista recoge, como tema de investigación, el estudio de la vinculación entre las características del entorno familiar con los resultados, ya sean de éxito o de fracaso escolar, que se logran en la escuela. Puntualizando los aspectos del entorno familiar que incurren sobre el rendimiento académico de los hijos, se ha considerado el papel de variables estructurales tales como el tamaño de la familia, el número de hijos, la composición de la familia, la estabilidad familiar, la profesión ejercida, el nivel de ingresos o los recursos materiales disponibles. De igual manera, es importante destacar las características de las familias, atendiendo a los valores, el estilo educativo de los padres, los estilos de vida y el clima relacional entre los miembros de la familia (Gimeno, 1984, p. 33).

En otras investigaciones realizadas en este mismo ámbito, se han analizado los aspectos culturales y la implicación de los padres con la escuela y la acción que se ejercen sobre los hijos. Han sido objeto de estudio variables como el nivel de 
estudios de los padres, el entorno en que vive la familia, las expectativas que se tienen sobre el nivel educativo, los estímulos que la familia proporciona a los hijos, la variedad de materiales educativos que pone a su disposición, la colaboración y supervisión de los padres sobre las tareas escolares realizadas en casa, el nivel de conocimientos de los padres sobre los contenidos escolares (Martínez y Álvarez, 2005, p. 64).

La mayoría de las variables han mostrado valores diferenciados cuando se analizan el éxito o el fracaso escolar en los niños. En una investigación realizada en la comunidad de Madrid, se analizó cómo un grupo de estudiantes de bachillerato obtuvo un alto rendimiento académico debido al compromiso de la escuela en dar una educación de calidad; además, se destacó la presencia de factores relativos a la educación familiar (Jiménez, 2006, p. 65). De igual manera, se han buscado empíricamente razones en las familias que aclaren el tema del fracaso escolar, aspectos estructurales y también aspectos dinámicos, como es el clima y entorno familiar (Ruiz, 2001, p. 29).

Si tomamos en cuenta las evidencias presentadas por la investigación acerca de la relación familia-escuela en el aprendizaje escolar de los hijos, podríamos respaldar la afirmación de Martínez (1992, p. 45) al reflexionar que el rendimiento, sea éxito o fracaso escolar, no debería constituir un asombro si se tiene un apropiado conocimiento de los condicionantes del entorno y del contexto familiar en el que los niños se desenvuelven.

En el contexto de una relación de colaboración entre la familia y la escuela, orientada al logro de unos objetivos educativos y a un aprendizaje en común, las características estructurales y de funcionamiento de la familia, y el ambiente educativo que se lleve en el hogar, son factores que influyen evidentemente en el éxito o fracaso escolar (Ruiz y García, 2001, p. 53).

\section{Cuerpo de la reflexión}

Al analizar la interrelación de la familia con la escuela, es importante tener presentes los roles que ejerce cada uno de los miembros y la afinidad que se ha de dar entre ellos, con el fin de igualar el patrón que ha de servir de guía en la socialización y formación del hijo que ha iniciado ya un proceso intencional e informal y que ahora viene a ser complementado por la escuela en forma ordenada e institucionalizada.

En el texto de Martiña (2003, p. 35) se perciben a la escuela y la familia como las dos instituciones que a lo largo de los siglos se han encargado de criar, socializar y preparar a las nuevas generaciones para insertarse positivamente en el mundo social y cultural de los adultos. Es decir que se hace necesaria una interrelación familia-escuela con el fin de lograr el objetivo de brindar orientación acorde a las necesidades del estudiante, en donde el manejo de variables 
conduzca a dar respuesta a un sujeto que está en proceso de desarrollo de su personalidad y madurez.

Tanto la escuela como la familia deben formar en valores, pues estos son componentes necesarios para la realización del ser humano y para su ubicación en un medio social. Según los resultados obtenidos a partir de entrevistas realizadas a estudiantes de grados quinto, sexto y séptimo, vemos que ellos perciben algunos principios en la relación familia-escuela y los hemos interpretado así:

Ante la pregunta ¿para ti qué relación hay entre familia y escuela? Así responde un estudiante: "en la familia lo educan y en la escuela aprende uno cosas". La familia es la primera responsable de la educación de los hijos y debe ser ella el primer sujeto-objeto de su formación.

¿Qué nivel de importancia le dan tus padres a tu educación? Un estudiante responde: "mi mamá sí le da importancia a las cosas del colegio, al acompañamiento de mis tareas, a las citaciones y todo eso". Los padres deben dar respuesta urgente a la necesidad del momento presente, deben ser agentes activos en la educación de sus hijos y es el objetivo, dentro de los propósitos globales, acompañarlos en el proceso.

Ante el cuestionamiento ¿cómo crees que es la comunicación entre docentes y padres de familia y por qué? Los estudiantes responden: "sí hay buena comunicación y en ocasiones mi mamá llama a los profesores para ver cómo voy", otro menciona: "mi mamá, siempre que necesita hablar con los profesores, les manda una nota y habla con la profesora". Las actuales circunstancias hacen evidente la importancia de aumentar la integración de los padres con el colegio, ofreciéndoles campos dirigidos hacia la participación y el diálogo.

Propiciar la formación de una nueva familia, centrada en la educación de los valores y formas de respeto a la vida y a los derechos de los demás miembros de la sociedad. Menciona el estudiante: "educarse es como tener valores y ser una buena persona".

En el mundo de cada familia se encuentra que los padres si están preocupados, porque no tienen los elementos fundamentales para dar la educación y orientación adecuadas a sus hijos. Al plantearse cómo acompañar a los hijos en la tarea de formarse en actitudes positivas que faciliten el proceso, los padres saben, en algunos casos, que deben modificar las formas de exigir, ordenar y enseñar a los hijos, pero no saben cómo hacerlo.

La pedagogía actual debe ser integradora y debe asumir el entorno familiar, con la respectiva participación de los padres e hijos en el proceso formativo de cara al presente, lo que demanda profundos e innovadores cambios, como urgencia para adaptarse a la cultura. 
El pretender un acercamiento entre la familia y la escuela no es tarea fácil, por cuanto hay una historia de años atrás de un modelo tradicional, donde la autoridad desciende en forma vertical. El posmodernismo, por sus muchas razones de cambio e inestabilidad, suscita el acercamiento de los padres a los hijos, en las formas más inesperadas, a través del diálogo, la autonomía, la corresponsabilidad y la democracia como necesidades prioritarias.

\section{Importancia del apoyo de los padres en las actividades escolares de sus hijos}

El apoyo de los padres en las actividades escolares de los hijos se expresa proporcionando no solo los elementos y las condiciones físicas para el desarrollo del estudio, sino también permitiendo que los niños asuman su responsabilidad en la realización de los deberes académicos. En ocasiones, algunos padres confunden el apoyo escolar a los hijos con algunos tipos de proteccionismo, como hacerles los trabajos y las tareas. Desde otra perspectiva, este apoyo suele asumirse sentando al lado del niño y supervisando que haga su tarea. "Los adultos pueden estar cerca para observar que los niños hagan la tarea sin contratiempos, y habrá ocasiones en las cuales sea necesario ayudarles, pero no es lo mismo ayudarles que hacerles la tarea" (Martiña, 2003, p.30). Lo conveniente es revisar el resultado y si el niño no entiende, sentarse y darle la explicación pertinente, pero, sobre todo, permitir que él asuma la responsabilidad de su deber.

Dicha participación puede ser de doble vía: por una parte, los padres ayudan a los profesores y a los estudiantes en las actividades escolares que se consideran convenientes, con asesoramiento de la escuela, para el aprendizaje en el hogar. Y se dan sugerencias para que los padres ayuden en casa a sus hijos en actividades coordinadas con la enseñanza recibida en el aula.

Sin embargo, la separación entre la escuela y la vida cotidiana sigue siendo un problema para la educación. Si los profesores desconocen las condiciones y peculiaridades del contexto de la educación familiar, difícilmente podrán incorporar el contexto sociofamiliar a sus proyectos educativos y curriculares, es por esto que dentro de esta investigación se busca analizar las percepciones que tienen los estudiantes desde los imaginarios que tienen sobre la escuela y su relación con la familia, ya que, a través del estudio, se puede evidenciar que el vínculo entre las dos instituciones contribuye en el desempeño de los educandos del ciclo III. Las expectativas de las familias frente a la escuela afirman que estas pueden entender la escuela como un lugar de aparcamiento de los hijos, como un lugar de adquisición de conocimientos con sentido utilitario o como un lugar de formación integral.

Algunas investigaciones han comprobado que la participación plena de los padres en la escuela es un factor importante para el éxito académico de sus hijos. Los padres constituyen un recurso frecuentemente oculto por la escuela y es importante que los profesores reconozcan su potencial. 
La investigación demuestra que, en la mayoría de ocasiones, la relación escuelafamilia es muy limitada, de tal manera que se reducen las reuniones de padres a entrega de boletines, dar avisos, muchas veces regaños sobre la asistencia del estudiante, el porte del uniforme, el aseo, el bajo rendimiento académico, la indisciplina, entre otros. A veces nos parece que la pedagogía es para las clases, pero no para las reuniones, y estas reuniones de padres también necesitan formas pedagógicas, tal vez unas distintas. Sin embargo, las llamadas a los padres que tienen niños con problemas académicos o disciplinarios no pueden seguir siendo un tormento para el estudiante, así lo afirman los estudiantes del ciclo III ante la pregunta ¿han sido citados tus acudientes por alguna persona diferente de tu director de grupo? (Sí, no y por qué), responde un estudiante: "a mí me ha mandado citas la profesora y la coordinadora por cuestiones disciplinarias", otro estudiante dice: "la coordinadora y el profesor de música por problemas académicos".

La preocupación de esa relación empieza con aclarar la función de los docentes dentro de la comunidad. El repertorio de relaciones con el contexto es pobre y rutinario, necesitamos arriesgarnos a crear nuevas herramientas y renovar muchas de nuestras ideas para aportar a la construcción de las comunidades educativas. Es necesario desarrollar las instancias mediadoras entre las familias y la escuela, pues la integración de la escuela con la familia supone la aceptación del conflicto y de la diversidad. La relación de la escuela con los padres y madres y con las demás personas de la comunidad debe contemplar la necesidad de la diferencia y la posibilidad de la controversia. Por lo tanto, la familia y la escuela tienen un rol fundamental en la educación de los menores, sobre todo porque la función conjunta entre los dos permite un adecuado desarrollo integral de los estudiantes, debido a que lo que se transmite en la escuela incide en las dinámicas familiares al interior del hogar, y lo que allí se genera incidirá en el desempeño académico de los estudiantes. En este sentido, es una relación directa, ya que si una mejora, la otra también lo hará y viceversa.

Actualmente se le exige a la escuela la resolución de múltiples problemas de la sociedad, como la violencia y la pérdida de valores, entre otros. Esto no ha hecho más que proponerle nuevas responsabilidades que la escuela no puede enfrentar sola. Para los docentes, pensar que la escuela no puede hacer nada ante esta nueva realidad se constituye en pesimismo pedagógico, pero, por otra parte, pensar que la escuela es mágica y puede resolver todos esos problemas es un sueño pedagógico. No pretendemos que la escuela articule por sí sola una sociedad tan fragmentada como en la que estamos actualmente. La escuela no puede plantearse la reconstrucción de la comunidad social, pero sí la construcción de una comunidad escolar viva que establezca relaciones educativas interdependientes con su contexto, es decir, que contribuya a la construcción de comunidades educativas. En 2006, Forest y Bacete sostuvieron que "las comunidades educativas, a menudo, deciden conjuntamente hacer cambios en el sistema que fomentarán la implicación de los padres y mejorarán la educación" (p. 43). Por lo tanto, se pretende llamar la atención acerca del hecho de que los padres se den cuenta de la ruptura relacional que se ha generado entre la familia y la escuela, pero que a través de la unión y el trabajo en equipo de las dos 
instituciones se pueden lograr cambios significativos en los procesos formativos de los menores, sobre todo en aquellos ciclos en los que han impactado los diferentes medios de comunicación, redes sociales y demás instrumentos que de una $u$ otra forma terminan convirtiéndose en las herramientas a las cuales acceden los jóvenes, dejando de lado la función que desempeña la familia.

Para algunos docentes es casi imposible imaginar su articulación con padres de familia y con personas de la comunidad-contexto, si antes no se ha dado un proceso de articulación de los docentes de la escuela. La participación no solo debe ser un instrumento al servicio de la gestión, sino un medio educativo. Lo que se ha dado es un modelo de participación separada y puntual que la mayoría de las veces se manifiesta y agota en la votación y en la delegación de responsabilidades.

Los ámbitos más apropiados para el ejercicio y aprendizaje de la participación son aquellos en los que se desarrolla la cotidianidad, esto es, los estudiantes en el aula, los padres en la relación tutorial, los profesores con sus colegas y directivos. En la vida concreta de las escuelas, la participación de los padres se ha convertido en un conflicto que, aparentemente, compromete la privacidad y el profesionalismo del trabajo de los educadores.

Participación y colaboración no significan trabajar juntos y en armonía. Se trata de un proceso lleno de conflictos, discusión y tensiones. Tampoco es posible pretender la uniformidad: la cultura de colaboración requiere un acuerdo básico sobre los valores educativos más importantes, pero también respeta la diversidad y tolera el desacuerdo. La participación familia-escuela debe estar dirigida específicamente al cambio de las relaciones de poder en la perspectiva de hacerlas más horizontales, más dialógicas y por consiguiente más democráticas. "La vida cotidiana de una escuela ofrece numerosas oportunidades favorables a la participación de todos. Tales experiencias entrañan un valor educativo extraordinario, pues, al tomar parte en un proyecto común, [...] gracias a los procesos comunicativos, se van aprendiendo recursos y formatos de actuación cultural" (SEP, 2009, p. 210).

Existen algunas condiciones, dentro y fuera de las escuelas, que ejercen un papel determinante con respecto a la escasa o nula integración de los padres con la institución educativa. Entre estas se encuentran: cultura de no participación, circunstancias económicas, sociales y culturales difíciles. Todos estos factores se conjugan, impidiendo la participación apropiada de las familias.

Otra causa asociada a la no participación son los contenidos de la educación escolar que implican una sola de las formas del saber existentes en la sociedad; los métodos de enseñanza fundamentados en la supuesta diferencia entre los que saben y los que no saben y la predominante memorización de contenidos, en vez de promover la creatividad, son componentes que adormecen la participación. El resultado se traduce en la aceptación por parte de los estudiantes en tomar posturas y actitudes totalmente pasivas. 
Son muchos los interrogantes que quedan pendientes, como ¿por qué siempre se ha señalado la relación escuela y la familia como una dificultad y no como una experiencia significativa donde se vean logros y aprendizajes positivos? Se trata de una posibilidad educativa con capacidad para conectar la educación y los procesos participativos con los referentes culturales del contexto. Se sustenta que la participación individual de los padres en la escuela puede resumirse en dos actividades principales.

En primer lugar, está la comunicación entre los padres y los profesores y directivos de la escuela, la cual, en buena lógica, parece muy conveniente para un buen funcionamiento del proceso educativo. En segundo lugar, está el apoyo que pueden prestar los padres a las actividades de la escuela, ya sea en forma de ayuda a los hijos a la hora de hacer los deberes, de tomarles la lección, de participar en actividades organizadas por el centro o de coordinar con éste la realización de actividades educativas con los hijos en el tiempo de ocio. (Pérez, Rodríguez, y Ferrer, 2001, p.67)

Algunos planteamientos educativos y de trabajo con la comunidad analizan las diferencias, la diversidad, como sinónimos de problema. La importancia de este planteamiento radica en que permite el estudio y la validación de saberes construidos en las prácticas sociales de la relación familia-escuela. El diálogo de saberes y la negociación cultural se convierten en una herramienta para la reconstrucción de los saberes de las personas, para su enriquecimiento y complejización.

\section{Metodología}

La investigación se desarrolló con base en una encuesta y en entrevistas semiestructuradas que se realizaron a 48 estudiantes del ciclo III de la educación básica de tres colegios públicos de la localidad de Kennedy, en Bogotá. La encuesta se enfoca en develar las diferentes tendencias sobre las percepciones que tienen los estudiantes con respecto a las principales características de la relación que se da entre familia y escuela. Las entrevistas permiten indagar de forma profunda cómo se presentan las relaciones, abriendo la posibilidad de que los entrevistados describan, expliquen y argumenten las razones de los complejos hechos que se presentan en la interacción escuela y familia, datos que se organizan tanto desde las categorías previstas como desde las emergentes.

\section{Resultados y conclusiones}

Los resultados muestran las características de la relación familia-escuela evidenciada desde la percepción que tienen los estudiantes indagados. La estructura de las familias de los estudiantes entrevistados se enmarca en el núcleo de la 
familia tradicional, cobijados por las presencias materna y paterna, además de la compañía de sus hermanos, lo que quiere decir, aparentemente, que tienen un acompañamiento afectivo, económico y apoyo para su desempeño escolar. Otra tendencia a resaltar la constituye la existencia de las madres cabeza de hogar, pues otro segmento de los estudiantes convive en una familia cuya responsable es solamente la madre de familia. También se encuentran familias reconstituidas donde hay un padrastro o una madrastra. Solo algunos de los entrevistados no tienen una familia definida, al parecer, viven en una familia extensa y no están bajo el cuidado de una figura materna o paterna.

La mayoría de los padres, casi un 70\%, cursó bachillerato, lo que significa que aunque no poseen un nivel alto de estudios, sí tienen unas fuentes académicas básicas para realizar el acompañamiento escolar a sus hijos; sin embargo, dicho apoyo escolar poco se ve reflejado en las familias, lo que conlleva al planteamiento de profundizar en el conocimiento de lo que sucede en el contexto de cada hogar.

Los datos suministrados por los estudiantes permiten deducir que en los hogares en donde se acompaña en las tareas a los hijos, en su gran mayoría, son los padres de familia las personas que por excelencia apoyan el proceso educativo de los menores y en un pequeño rango los hermanos mayores acompañan este proceso.

Con respecto a las razones que esgrimen algunos estudiantes para no contar con la colaboración de sus padres en la realización de tareas escolares, refieren que el tiempo dedicado al trabajo y la falta de vínculos entre padres e hijos se constituyen en factores que distancian a los progenitores de sus hogares.

Los entrevistados plantean, de manera general, que los encuentros entre familiares y docentes siempre favorecen el desempeño académico. Así, debe ser motivo de satisfacción para la institución educativa el hecho de que los estudiantes identifiquen este logro, lo que a su vez representa un avance en la tarea de la institución escolar y un reconocimiento sincero, venido de los niños, centro del quehacer docente.

Con base en el anterior recorrido, se puede afirmar que la relación escuela-familia es un proceso bidireccional que requiere un trabajo en equipo generado desde aula, desde las necesidades, requerimientos y conocimientos de los niños y jóvenes, que se van articulando y fortaleciendo al interior de todos los estamentos de la comunidad educativa. Es allí y en el hogar donde se edifican, se viven y se apropian los derechos humanos y, por ende, los de los niños.

Los conceptos "familia" y "escuela" nos permiten entrar a considerar a toda la comunidad educativa y sus relaciones con el contexto, los padres, el barrio, la ciudad. La interacción en el ambiente educativo implica poseer una gran valoración, perder el miedo a la transformación y el cambio y darse cuenta de que debemos ser menos transmisores de información y más aquellos docentes que posibiliten herramientas creativas, innovadoras y de reflexión. 
Con relación a las familias, se debe tener en cuenta el espacio geográfico en que se encuentran, al igual que las características económicas, sociopolíticas y culturales específicas que las envuelven. La realidad que nos muestra la investigación es que en los tres colegios distritales ubicados en la localidad de Kennedy, en Bogotá, hay estudiantes que proceden de lugares totalmente distantes y ajenos y con características disimiles. Dentro de la investigación se evidenciaron algunos aspectos que influyen en el rendimiento de los estudiantes, encontramos aspectos ligados a la composición de la familia, el nivel de estudios, la profesión ejercida por los padres, así como el acompañamiento y supervisión de los padres en las tareas escolares realizadas en casa.

En la dinámica social es necesario tener en cuenta estos núcleos: la familia, la escuela, los grupos de estudiantes y la comunidad del contexto. La escuela debe ser mirada y analizada como una institución diferente a todas las demás instituciones de nuestra sociedad: si es comunidad de saberes tiene que hablarse del proceso de enseñanza y aprendizaje, allí adquieren sentido el conocimiento, la ciencia y la investigación. En la escuela compartimos experiencias y construimos un orden social con un conjunto de seres que hacen la diferencia. Entonces, desde ese punto de vista, es fácil entender la escuela, buscar en ella y enamorarse de ella en un espacio para el desarrollo social.

La relación familia-escuela hoy cobra nuevos sentidos y significados, pero sobre todo presenta nuevos retos. Del análisis extractado surge una propuesta alternativa de indagación; consistente en analizar la relación familia-escuela y verla en un sentido de proyección conjunta, considerándola como recontextualizadora de la cultura y como posibilitadora de nuevos horizontes y ventanas del conocimiento de usos y costumbres. La escuela puede servir de catalizador en la construcción de espacios para la democracia, especialmente para aquellos niños y jóvenes procedentes de hogares violentos y conflictivos. La institución educativa enfrenta hoy el reto de construir soluciones para que los niños, niñas y jóvenes conozcan otras formas de relaciones humanas con la familia y la sociedad. Visto esto, la misión formativa no puede, por tanto, permanecer al margen de esta realidad.

\section{$\mathbf{R}_{\text {eferencias }}$}

Bourdieu, P., y Passeron, J. C. (1990). Reproduction in education, society and culture (Vol. 4). Newcastle: Sage.

Díez, J. J. (1998). Familia-escuela: una relación vital. Madrid: Narcea.

Fernández, C. J. (2006). Educación familiar y alumnos con alto rendimiento. Revista española de pedagogía,64(234), 273-299.

Forest, C., y Bacete, F. J. G. (2006). Comunicación cooperativa entre la familia y la escuela (Vol. 1). Valencia: Nau Llibres. 
García, F. C. (1997). Ideas de los padres respecto al aprendizaje y rendimiento escolar. Revista de Psicología de la Educación (22), 23-35.

Garreta, J. (Ed.). (2007). Relación familia-escuela. Lleida: Universitat de Lleida.

Gimeno, J. B. (1984). El fracaso escolar en la enseñanza primaria: medios para combatirlo: Estudio comparativo internacional. París: Unesco.

González, R. A. M. (1992). Factores familiares que intervienen en el progreso académico de los alumnos. Aula abierta, (60), 23-40.

Marchesi, Á, y Martín, E. (1998). Calidad de la enseñanza en tiempos de cambio. Madrid: Alianza editorial.

Martínez González, R. A., y Álvarez Blanco, L. (2005). Fracaso y abandono escolar en Educación Secundaria Obligatoria: implicación de la familia y los centros escolares. Aula Abierta, 85, 127-146.

Martiñá, R. (2003). Escuela y familia: una alianza necesaria. México: Editorial Pax.

Molero, D. (2003). Estudio sobre la implicación de las familias en los procesos de enseñanza y aprendizaje. Revista Española de Orientación y Psicopedagogía, 14 (1), 61-82.

Ordóñez Sierra, R. (2005). Medios para mejorar la relación entre la familia y la escuela. Kikiriki. Cooperación educativa (78), 38-42.

Pérez-Díaz, V., Rodríguez, J. C., y Ferrer, L. S. (2001). La familia española ante la educación de sus hijos. Madrid: Fundación" la Caixa".

Ruiz de Miguel, C. R. (2001). Factores familiares vinculados al bajo rendimiento. Revista complutense de educación, 12(1), 81-113.

Ruiz, C., y García, M. (2001). Propuesta de un modelo estructural de la influencia familiar en los resultados escolares del niño. En Aidipe (Comp.), X Congreso Nacional de Modelos de Investigación Educativa (pp. 93-98).

Sarramona López, J. (1999). La educación en la familia y en la escuela. Madrid: Educar.

Secretaría de Educación Pública de México (2009). Antología de Gestión Escolar. Programa Nacional de Carrera Magisterial. México: SEP.

Vila, I. (1998). Familia, escuela y comunidad. Barcelona: ICE Universitat Barcelona. 